\title{
Ground state solutions for a class of quasilinear Choquard equation with critical growth
}

\author{
Liuyang Shao ${ }^{\mathrm{a}}$, Haibo Chen ${ }^{\mathrm{b}}$, Yingmin Wang ${ }^{\mathrm{a}}$ \\ a School of Mathematics and Statistics, GuiZhou University of Finance and Economics, Guiyang, Guizhou 550025, P. R. China. \\ ${ }^{b}$ School of Mathematics and Statistics, Central South University, Changsha, Hunan, 410083, P. R. China.
}

\begin{abstract}
In this paper, we consider the following quasilinear Choquard equation with critical nonlinearity

$$
\begin{cases}-\Delta u+V(x) u-u \Delta u^{2}=\left(I_{\alpha} *|u|^{p}\right)|u|^{p-2} u+u^{2\left(2^{*}\right)-2} u, & x \in \mathbb{R}^{N}, \\ u>0, & x \in \mathbb{R}^{N},\end{cases}
$$

where $I_{\alpha}$ is a Riesz potential, $0<\alpha<N$, and $\frac{N+\alpha}{N}<p<\frac{N+\alpha}{N-2}$, with $2^{*}=\frac{2 N}{N-2}$. Under suitable assumption on $V$, we research the existence of positive ground state solutions of above equations. Moreover, we consider the ground state solution of the equation (1.4). Our work supplements many existing partial results in the literature.
\end{abstract}

Keywords: Quasilinear equation, variational methods, ground state solution, Choquard type.

2020 MSC: 35B09, 35J20.

(C)2021 All rights reserved.

\section{Introduction and main results}

In present paper, we investigate the following quasilinear Choquard equation with critical growth

$$
\begin{cases}-\Delta u+V(x) u-u \Delta u^{2}=\left(I_{\alpha} *|u|^{p}\right)|u|^{p-2} u+u^{2\left(2^{*}\right)-2} u, & x \in \mathbb{R}^{N}, \\ u \in H^{1}\left(\mathbb{R}^{N}\right), & x \in \mathbb{R}^{N},\end{cases}
$$

where $I_{\alpha}$ is a Riesz potential, $0<\alpha<N$, and $\frac{N+\alpha}{N}<p<\frac{N+\alpha}{N-2}$. Quasilinear Schrödinger equations of the form

$$
i \partial_{\mathrm{t}} z=-\triangle z+V(x) z-f\left(|z|^{2}\right) z-\triangle h\left(|z|^{2}\right) h^{\prime}\left(|z|^{2}\right) z,
$$

have been derived as models of several physical phenomena. Here $V=V(x), x \in \mathbb{R}^{N}$, is a given potential, $K, V$ are real functions. For instance in the case $h(s)=s$, we obtain

$$
i \partial_{\mathrm{t}} z=-\triangle z+\mathrm{V}(\mathrm{x}) z-\mathrm{f}\left(|z|^{2}\right) z-\left(\triangle|z|^{2}\right) z
$$

\footnotetext{
*Corresponding author

Email addresses: sliuyang316@163.com (Liuyang Shao), chb_math@163.com (Haibo Chen), 1017783171@qq.com (Yingmin Wang)
}

doi: $10.22436 /$ jnsa.014.06.02

Received: 2021-02-06 Revised: 2021-02-27 Accepted: 2021-03-18 
which has been called the superfluid film equation in plasma physics by Kuriharn in [16] (cf. [17, 18]), In the case $h(s)=(1+s)^{\frac{1}{2}}$, equation (1.2) models the self-channeling of a high-power ultra short laser in mater, see [4,12] and the references in [10]. Equation (1.2) also appears in plasma physics and fluid mechanics [16, 17, 21, 26], the theory of Heisenberg ferromagnets and magnons [3], dissipative quantum mechanics, and condensed matter theory [13, 22]. For more details, we refer the readers to [19] and the references therein.

In recent years, the study on the quasilinear Schrödinger equation (1.2) is always a topic of great interest, mathematicians have established several methods to treat Eq. (1.3), for example, the dual approach, the perturbation method, and the Nehari method. See for instance $[1,6,9,11,14,23-25,27-30,32]$, and the references therein. However, the problem (1.1) with Choquard type nonlinearity has only been studied in $[2,7,8,33]$.

It's remarkable that there are few papers investigating quasilinear Choquard equation with critical growth. In [7], they study the following Choquard type quasilinear Schrödinger equation

$$
-\triangle \mathrm{u}+\mathrm{V}(\mathrm{x}) \mathrm{u}-\triangle\left(\mathrm{u}^{2}\right) \mathrm{u}=\left(\mathrm{I}_{\alpha} *|\mathrm{u}|^{\mathrm{p}}\right)|\mathrm{u}|^{\mathrm{p}-2} \mathrm{u}, \quad x \in \mathbb{R}^{\mathrm{N}},
$$

where $N \geqslant 3,0<\alpha<N, \frac{2(N+\alpha)}{N}<p<\frac{2(N+\alpha)}{N-2}, V: \mathbb{R}^{N} \rightarrow \mathbb{R}$ is radial potential and $\mathrm{I}_{\alpha}$ is a Riesz potential. They consider the existence of ground state solutions. By the motivation of above work, In our article, we establish the existence of ground state solution for problem (1.1) with critical. Moreover, we are interested in the problem that how the potential $\mathrm{V}(\mathrm{x})$ affect the existence of ground state solution for the problem (1.1). Since the nonlinearity of problem (1.1) is nonlocal, it is much more difficult to obtain the existence of ground solutions.

Before stating our main result, we suppose that the functions $V(x)$ satisfy the following assumptions:

$\left(V_{1}\right) V \in C\left(\mathbb{R}^{N}\right)$ satisfies $\inf _{x \in \mathbb{R}^{N}} V(x)>V_{0}>0$, where $V_{0}$ is a constant;

$\left(V_{2}\right)$ meas $\left\{x \in \mathbb{R}^{N}:-\infty<V(x) \leqslant \mu\right\}<+\infty$ for all $\mu \in \mathbb{R}$.

Now we state our main results as follows.

Theorem 1.1. Suppose that $0<\alpha<\mathrm{N}, \frac{\mathrm{N}+\alpha}{\mathrm{N}}<\mathrm{p}<\frac{\mathrm{N}+\alpha}{\mathrm{N}-2}$, and $\left(\mathrm{V}_{1}\right),\left(\mathrm{V}_{2}\right)$ hold, then equation (1.1) admits a ground state solution in $\mathrm{E}$.

Next, we study the ground state solution of the following equation.

$$
\begin{cases}-\triangle \mathfrak{u}+V(x) u-u \Delta u^{2}=\left(I_{\alpha} *|u|^{p}\right)|u|^{p-2} u+u^{q-2} u, & x \in \mathbb{R}^{N}, \\ u \in H^{1}\left(R^{N}\right), & x \in \mathbb{R}^{N},\end{cases}
$$

where $\frac{N+\alpha}{N}<p<\frac{N+\alpha}{N-2}, q \in\left(2,2^{*}\right)$.

Theorem 1.2. Let $\mathrm{N} \geqslant 3, \mathrm{q} \in\left(2,2^{*}\right)$ and $\mathrm{V} \in \mathrm{L}^{\infty}\left(\mathbb{R}^{\mathrm{N}}\right)$, if $\left(\mathrm{V}_{1}^{\prime}\right) \inf _{\mathrm{y} \in \mathbb{R}^{\mathrm{N}}} \mathrm{V}(\mathrm{y})>0$, and

$\left(\mathrm{V}^{\prime}{ }_{2}\right) \mathrm{V}(\mathrm{x}) \leqslant \lim _{|\mathrm{y}| \rightarrow \infty} \mathrm{V}(\mathrm{y})=\mathrm{V}_{\infty}<+\infty$ and the inequality is strict in a subset of positive Lebesgue measure,

then Eq. (1.4) admits a ground state solution.

Remark 1.3. Here we don't use the Pohozayev identity, and we have taken advantage of the monotonicity of perturbation and Nehari manifold method to obtain the ground state solutions.

Notation. In this paper we make use of the following notations: $C$ will denote various positive constants; the strong (respectively weak) convergence is denoted by $\rightarrow$ (respectively $\rightarrow$ ); o(1) denotes o(1) $\rightarrow 0$ as $n \rightarrow \infty, B_{\rho}(0)$ denotes a ball centered at the origin with radius $\rho>0$.

The remainder of this paper is organized as follows. In Section 2, some preliminary results are presented. In Section 3, we obtain that (1.1) and (1.4) have ground state solutions. 


\section{Variational setting and preliminaries}

To prove our conclusion, we give some basic notations and preliminaries. First, we can rewrite (1.1) as

$$
-\triangle \mathrm{u}+\mathrm{V}(\mathrm{x}) \mathrm{u}-\triangle\left(\mathrm{u}^{2}\right) \mathrm{u}=\mathrm{I}_{\alpha} *|\mathrm{u}|^{\mathrm{p}}|\mathrm{u}|^{\mathrm{p}-2} \mathrm{u}+|\mathrm{u}|^{2\left(2^{*}\right)-2} \mathrm{u}, \quad \text { in } \mathbb{R}^{\mathrm{N}} .
$$

It may also be noted that we can not apply directly the variational method to study (1.1), since the natural associated functional I given by

$$
\mathrm{I}(\mathrm{u})=\frac{1}{2} \int_{\mathbb{R}^{N}}\left(1+2 \mathrm{u}^{2}\right)|\nabla u|^{2} \mathrm{~d} x+\frac{1}{2} \int_{\mathbb{R}^{N}} \mathrm{~V}(x) \mathrm{u}^{2} \mathrm{~d} x-\frac{1}{2 p} \int_{\mathbb{R}^{N}} \mathrm{I}_{\alpha} *|\mathrm{u}|^{p}|u|^{p-1} u d x-\frac{1}{2\left(2^{*}\right)} \int_{\mathbb{R}^{N}} \mathrm{u}^{2\left(2^{*}\right)} \mathrm{udx}
$$

is not well defined in general. we make the changing of variables $w=f^{-1}(u)$, where $f$ is defined by: $f^{\prime}(t)=\frac{1}{\sqrt{1+2 f^{2}(t)}}$ on $[0, \infty)$ and $f(t)=f(-t)$ on $(-\infty, 0]$. If we make the change of variable $(u=f(w))$, we may rewrite equation $\mathrm{I}(\mathrm{u})$ in the form

$$
J(w)=\frac{1}{2} \int_{\mathbb{R}^{N}}\left(|\nabla w|^{2}+V(x) f^{2}(w)\right) \mathrm{d} x-\frac{1}{2 p} \int_{\mathbb{R}^{N}} I_{\alpha} *|f(w)|^{p}|f(w)|^{p-1} f(w) d x-\frac{1}{2\left(2^{*}\right)} \int_{\mathbb{R}^{N}}|f(w)|^{2\left(2^{*}\right)} \mathrm{d} x .
$$

It can be easily proved that the functional $\mathcal{J}(w)$ is of class $C^{1}$ (see [32]) in E. Moreover, the critical points of $\mathcal{J}$ are weak solutions of the equation

$$
\int_{\mathbb{R}^{N}}|\nabla w \nabla \varphi|+V(x) f(w) f^{\prime}(w) \varphi-\int_{\mathbb{R}^{N}}\left(I_{\alpha} *|f(w)|^{p}|f(w)|^{p-1} f^{\prime}(w) \varphi-\int_{\mathbb{R}^{N}} f^{2\left(2^{*}-2\right.}(w) f(w) f^{\prime}(w) \varphi=0 .\right.
$$

Be aware that if $w$ is a critical point of $\mathcal{J}$, then, $u=f(w)$ is a weak solution of the problem (1.1). For any $w \in \mathrm{E}$, let

$$
E=\left\{w \in H^{1}\left(\mathbb{R}^{N}\right) \mid \int_{\mathbb{R}^{N}} V(x) f^{2}(w) d x<\infty\right\}
$$

be a Hilbert space endowed with inner product and norm

$$
\|w\|_{\mathrm{E}}:=\langle w, w\rangle=\left(\int_{\mathbb{R}^{N}}\left(|\nabla w|^{2}+\mathrm{V}(x) w^{2}\right) \mathrm{d} x\right)^{\frac{1}{2}} .
$$

We denote by $\|\bullet\|_{p}$ the usual $L^{p}$-norm in the sequel for convenience, where $1 \leqslant p \leqslant+\infty$. In this step, we see that (1.1) is variational and its weak solutions are the critical points of the functional given by

$$
\mathcal{J}(w)=\frac{1}{2} \int_{\mathbb{R}^{N}}\left[|\nabla w|^{2}+V(x) f^{2}(w)\right] \mathrm{d} x-\left.\frac{1}{2 p} \int_{\mathbb{R}^{N}} \mathrm{I}_{\alpha} *|f(w)|\right|^{p}|\mathbf{f}(w)|^{p-1} f(w) \mathrm{d} x-\frac{1}{2\left(2^{*}\right)} \int_{\mathbb{R}^{N}}|f(w)|^{2\left(2^{*}\right)} \mathrm{d} x .
$$

Lemma 2.1 ([12]). The function $\mathrm{f}$ satisfies the following properties:

$\left(\mathrm{A}_{1}\right) \mathrm{f}$ is uniquely defined $\mathrm{C}^{\infty}$ function and invertible;

$\left(\mathrm{A}_{2}\right)\left|\mathrm{f}^{\prime}(\mathrm{s})\right| \leqslant 1$ and $|\mathrm{f}(\mathrm{s})| \leqslant|\mathrm{s}|$ for all $\mathrm{s} \in \mathbb{R}$;

$\left(\mathrm{A}_{3}\right) \frac{\mathrm{f}(\mathrm{s})}{\mathrm{s}} \rightarrow 1$ as $\mathrm{s} \rightarrow 0$;

$\left(\mathrm{A}_{4}\right) \frac{\mathrm{f}(\mathrm{s})}{\sqrt{\mathrm{s}}} \rightarrow 2^{\frac{1}{4}}$ as $\mathrm{s} \rightarrow \infty$;

$\left(A_{5}\right) \frac{f(s)}{2} \leqslant s f^{\prime}(s) \leqslant f(s)$ for all $s \geqslant 0$;

$\left(\mathrm{A}_{6}\right)|\mathrm{f}(\mathrm{s})| \leqslant 2^{\frac{1}{4}}|\mathrm{~s}|^{\frac{1}{2}}$ for all $\mathrm{s} \in \mathbb{R}$;

$\left(\mathrm{A}_{7}\right)$ the function $\mathrm{f}^{2}(\mathrm{~s})$ is strictly convex;

$\left(\mathrm{A}_{8}\right)$ there exists a positive constant $\mathrm{C}$ such that

$$
|f(s)| \geqslant \begin{cases}C|s|, & |s| \leqslant 1 \\ C|s|^{\frac{1}{2}}, & |s| \geqslant 1\end{cases}
$$


(A9) for each $\lambda>1$, we have $\mathrm{f}^{2}(\lambda \mathrm{s}) \leqslant \lambda \mathrm{f}^{2}(\mathrm{~s})$ for all $\mathrm{t} \in \mathbb{R}$;

$\left(\mathrm{A}_{10}\right)$ the function $\mathrm{f}^{-\mathrm{q}}(\mathrm{s}) \mathrm{f}^{\prime}(\mathrm{s})$ is strictly decreasing for $\mathrm{s}>0$ and $0<\mathrm{q}<1$;

$\left(\mathrm{A}_{11}\right)$ the function $\mathrm{f}^{\mathrm{q}}(\mathrm{s}) \mathrm{f}^{\prime}(\mathrm{s}) \mathrm{s}^{-1}$ is strictly increasing for $\mathrm{q} \geqslant 3$ and $\mathrm{s}>0$.

Now we recall the well know Hardy-Littlewood-Sobolev Inequality.

Proposition 2.2 ([15, Hardy-Littlewood-Sobolev inequality]). Let $t, r>1$ and $0<\mu<n$ with $\frac{1}{\mathrm{t}}+\frac{\mu}{n}+\frac{1}{r}, f \in$ $\mathrm{L}^{\mathrm{t}}\left(\mathbb{R}^{\mathrm{N}}\right)$ and $\mathrm{h} \in \mathrm{L}^{\mathrm{r}}\left(\mathbb{R}^{\mathrm{N}}\right)$. There exists a sharp constant $\mathrm{C}(\mathrm{t}, \mathrm{n}, \mu, \mathrm{r})$, independent of $\mathrm{f}, \mathrm{h}$ such that

$$
\int_{\mathbb{R}^{N}} \int_{\mathbb{R}^{N}} \frac{f(x) h(y)}{|x-y|^{\mu}} d x d y \leqslant C(t, n, \mu, r)\|f\|_{L^{t}\left(\mathbb{R}^{N}\right)}\|h\|_{L^{r}\left(\mathbb{R}^{N}\right)} .
$$

If $\mathrm{t}=\mathrm{r}=\frac{2 \mathrm{n}}{2 \mathrm{n}-\mu}$, then

$$
C(t, n, \mu, r)=C(n, \mu)=\pi^{\frac{\mu}{2}} \frac{\Gamma\left(\frac{n}{2}-\frac{\mu}{2}\right)}{\Gamma\left(n-\frac{\mu}{2}\right)}\left\{\frac{\Gamma\left(\frac{\mu}{2}\right)}{\Gamma(n)}\right\}^{-1+\frac{\mu}{n}} .
$$

In this case there is equality in (2.1) if and only if $\mathrm{f}=$ (constant)h and

$$
h(x)=A\left(r^{2}+|x-a|^{2}\right)^{-\frac{2 n-\mu}{2}}
$$

for some $\mathrm{A} \in \mathrm{C} .0 \neq \mathrm{r} \in \mathrm{R}$ and $\mathrm{a} \in \mathbb{R}^{\mathrm{N}}$.

Applying the Hardy-Littlewood-Sobolev inequality above and the Sobolev embedding theorem, we have

$$
\int_{\mathbb{R}^{N}}\left(I_{\alpha} *|u|^{p}\right)|u|^{p} \leqslant C\left(\int_{\mathbb{R}^{N}}|u|^{\frac{2 N p}{N+\alpha}}\right)^{\frac{N+\alpha}{N}} \leqslant C\|u\|^{p}
$$

for any $p \in\left(\frac{N+\alpha}{N}, \frac{N+\alpha}{N-2}\right)$, where $C>0$ is a constant depending on $N, \alpha$ and $p$.

\section{Proof of Theorem 1.1}

The functional $\mathcal{J}_{\geqslant}(w)$ satisfies the mountain pass geometry. Consider the set $S(\rho)=\{w \in E: \Phi(w)=$ $\left.\rho^{2}\right\}$, where $\Phi: E \rightarrow \mathbb{R}$ is given by

$$
\Phi(w)=\int_{\mathbb{R}^{\mathbb{N}}}\left(|\nabla w|^{2}+\mathrm{V}(x) \mathrm{f}^{2}(w)\right) \mathrm{d} x,
$$

since $\Phi(w)$ is continuous, then $S(\rho)$ is a closed subset and disconnects the space $E$ for $\rho>0$.

Lemma 3.1. There exist $\rho$ and $m$ such that $\mathcal{J}(w) \geqslant \alpha$ when $\int_{\mathbb{R}^{\mathbb{N}}}\left(|\nabla w|^{2}+V(x) f^{2}(w)\right) d x=\rho, w \in S(\rho)$.

Proof. By Lemma 2.1, $\left(A_{2}\right)$, and (2.1), we get that

$$
\begin{aligned}
& \mathcal{J}(w)=\frac{1}{2} \int_{\mathbb{R}^{N}}\left(|\nabla w|^{2}+\mathrm{V}(x) \mathrm{f}^{2}(w)\right) \mathrm{d} x-\frac{1}{2 p} \int_{\mathbb{R}^{N}}\left(\mathrm{I}_{\alpha} *|\mathrm{f}(w)|^{p}\right)|\mathrm{f}(w)|^{p} \mathrm{~d} x-\frac{1}{22^{*}} \int_{\mathbb{R}^{N}}|\mathrm{f}(w)|^{22^{*}} \\
& \geqslant \frac{1}{2} \int_{\mathbb{R}^{N}}\left(|\nabla w|^{2}+\mathrm{V}(x) f^{2}(w)\right) \mathrm{d} x--\frac{1}{2 p} \int_{\mathbb{R}^{N}}\left(I_{\alpha} *|f(w)|^{p}\right)|f(w)|^{p} \mathrm{~d} x-C\left(\int_{\mathbb{R}^{N}}|\nabla w|^{2} \mathrm{~d} x\right)^{\frac{2^{*}}{2}} \\
& \geqslant \frac{1}{2} \rho^{2}-\mathrm{C}_{0} \rho^{2 p}-\mathrm{C}_{1} \rho^{2^{*}}
\end{aligned}
$$

for small $\varepsilon>0$, where $C_{0}, C_{1}$ are constant. Choosing $\rho>0$ with $\frac{1}{2} \rho^{2}-C_{0} \rho^{2 p}-C_{1} \rho^{2^{*}}=\alpha>0$, then $\mathcal{J}(w) \geqslant \alpha$ for all $w \in S(\rho)$. We complete the proof.

Lemma 3.2. There exists $\|e\|>\rho$, with $\mathcal{\partial} \geqslant(w)<0$. 
Proof.

$$
\begin{aligned}
& \mathcal{J}(\mathrm{t} w) \leqslant \frac{\mathrm{t}^{2}}{2} \int_{\mathbb{R}^{N}}|\nabla w|^{2}+\mathrm{V}(\mathrm{x}) w^{2} \mathrm{~d} x-\frac{\mathrm{t}^{2 p}}{2 \mathrm{p}} \int_{\mathbb{R}^{N}}\left(\mathrm{I}_{\alpha} *|\mathrm{f}(w)|^{\mathrm{p}}\right)|\mathrm{f}(w)|^{\mathrm{p}} \mathrm{d} x-\frac{1}{22^{*}} \int_{\mathbb{R}^{N}}|\mathrm{f}(\mathrm{t} w)|^{22^{*}} \mathrm{~d} x \\
& \leqslant \frac{t^{2}}{2} \int_{\mathbb{R}^{N}}\left(|\nabla w|^{2}+V(x) w^{2}\right) d x-\frac{t^{2 p}}{2 p} \int_{\mathbb{R}^{N}}\left(I_{\alpha} *|f(w)|^{p}\right)|f(w)|^{p} d x-C t^{2^{*}} \int_{\mathbb{R}^{N}} w^{22^{*}} d x \\
& \rightarrow-\infty
\end{aligned}
$$

as $t \rightarrow+\infty$. This implies that there exists $\|e\|>\rho$, such that $\mathcal{J}<0$. The proof is completed.

Define

$$
\Gamma=\left\{\gamma \in \mathrm{C}^{1}([0,1], \mathrm{E}) \mid \gamma(0)=0, \gamma(1)=e\right\}
$$

Then

$$
c:=\inf _{\gamma \in \Gamma} \max _{t \in[0,1]} \mathrm{I}(\gamma(\mathrm{t}))
$$

is a critical value of $I(u)$.

Lemma 3.3. Suppose that the condition (V) hold. If $\left\{w_{n}\right\} \subset E$ is such that $\mathcal{J}\left(w_{n}\right) \rightarrow c$ and $\mathcal{J}^{\prime}\left(w_{n}\right) \rightarrow 0$, then $\left\{w_{\mathrm{n}}\right\}$ is bound in $\mathrm{E}$.

Proof. By $\left\{w_{n}\right\} \subset \mathrm{E}$ and $\mathcal{J}\left(w_{n}\right) \rightarrow c, \mathcal{J}^{\prime}\left(w_{n}\right) \rightarrow 0$, we have

$$
\begin{aligned}
\mathcal{J}\left(w_{n}\right)= & \frac{1}{2} \int_{\mathbb{R}^{N}}\left|\nabla w_{n}\right|^{2} \mathrm{~d} x+\frac{1}{2} \int_{\mathbb{R}^{N}} \mathrm{~V}(x) f^{2}\left(w_{n}\right) \mathrm{d} x \\
& -\frac{1}{2 p} \int_{\mathbb{R}^{N}} I_{\alpha} * \mid f\left(\left.w_{n}\right|^{p} \mid f\left(\left.w_{n}\right|^{p} \mathrm{~d} x-\frac{1}{22^{*}} \int_{\mathbb{R}^{N}} \mid f\left(\left.w_{n}\right|^{22^{*}} \mathrm{~d} x .\right.\right.\right.
\end{aligned}
$$

Thus for any $\varphi \in \mathrm{C}_{0}^{\infty}\left(\mathbb{R}^{\mathrm{N}}\right)$

$$
\begin{aligned}
g^{\prime}\left(w_{n}\right) \varphi= & \int_{\mathbb{R}^{N}}\left|\nabla w_{n} \nabla \varphi\right|+V(x) f\left(w_{n}\right) f^{\prime}\left(w_{n}\right) \varphi-\int_{\mathbb{R}^{N}} I_{\alpha} *\left|f\left(w_{n}\right)\right|^{p}\left|f\left(w_{n}\right)\right|^{p-2} f\left(w_{n}\right) f^{\prime}\left(w_{n}\right) \mathrm{d} x \\
& -\int_{\mathbb{R}^{N}} \mid f\left(\left.w_{n}\right|^{22^{*}-2} f\left(w_{n}\right) f^{\prime}\left(w_{n}\right)=o(1)\|\varphi\|_{E} .\right.
\end{aligned}
$$

It's remarkable that $\frac{f(t)}{f^{\prime}(t)} \rightarrow 0$ as $t \rightarrow 0$. We get $\frac{f(t)}{f^{\prime}(t)} \in E$ by direct computation moreover since $C_{0}^{\infty}\left(\mathbb{R}^{N}\right)$ is dense in E. We may choose $\varphi=\frac{f\left(w_{n}\right)}{f^{\prime}\left(w_{n}\right)}$ as test functions and obtain

$$
\left\langle\mathcal{J}^{\prime}\left(w_{n}\right), \varphi\right\rangle=\int_{\mathbb{R}^{N}}\left|\nabla w_{n}\right|^{2} \mathrm{~d} x+\int_{\mathbb{R}^{N}} \mathrm{~V}(x) \mathrm{f}^{2}\left(w_{n}\right) \mathrm{d} x-\int_{\mathbb{R}^{N}} \mathrm{I}_{\alpha} *\left|f\left(w_{n}\right)\right|^{p}\left|f\left(w_{n}\right)\right|^{p} \mathrm{~d} x-\int_{\mathbb{R}^{N}}\left|f\left(w_{n}\right)\right|^{22^{*}} \mathrm{~d} x .
$$

Thus it can be seen that

$$
\begin{aligned}
c+o(1)\left\|w_{n}\right\|_{E} & =\mathcal{J}\left(w_{n}\right)-\frac{1}{2 p}\left\langle\mathcal{J}^{\prime}\left(w_{n}\right), \varphi\right\rangle \\
& \geqslant\left(\frac{1}{2}-\frac{1}{2 p}\right) \int_{\mathbb{R}^{N}}\left(\left|\nabla w_{n}\right|^{2}+V(x)\left|f\left(w_{n}\right)\right|^{2}\right) d x+\left(\frac{1}{2 p}-\frac{1}{22^{*}}\right) \int_{\mathbb{R}^{N}}\left|f\left(w_{n}\right)\right|^{22^{*}} \mathrm{~d} x .
\end{aligned}
$$

Hence, We get $\left\{w_{n}\right\}$ is bounded in E. It is noteworthy that $\left|\nabla f\left(w_{n}\right)\right| \leqslant\left|\nabla w_{n}\right|$, it is concluded that $\left\{f\left(w_{n}\right)\right\}$ is also bounded in $\mathrm{E}$. The proof is completed.

Lemma 3.4. For each $\tau>0$, and the condition $(\mathrm{V})$ holding, then there exists $w \in \mathrm{H}^{1}\left(\mathbb{R}^{\mathrm{N}}\right)$ such that $\max _{\mathrm{t} \geqslant 0} \mathcal{J}\left(\mathrm{t} v_{\mathcal{\varepsilon}}\right) \leqslant$ $\frac{1}{2 N} S^{\frac{N}{2}}$, where $S:=\inf _{u \in D^{1,2}\left(\mathbb{R}^{N}\right)} \frac{\int_{\mathbb{R}^{N}}|\nabla w|^{2} \mathrm{dx}}{\left(\int_{\mathbb{R}^{N}}|w|^{2^{*} \mathrm{~d} x}\right)^{\frac{2}{2^{*}}}}$. 
Proof. Let $\phi \in \mathrm{C}_{0}^{\infty}\left(\mathrm{R}^{\mathrm{N}},[0,1]\right)$, and the possible candidate for $w$ is $v_{\varepsilon}=w_{\varepsilon} \phi$ where $\phi$ is a smooth cut-off function such that $\phi(x)=1$ if $|x| \leqslant 1, \phi(x)=0$ if $|x| \geqslant 2$ and $|\nabla \phi| \leqslant 2$ and the function $w_{\varepsilon}$ defined $w_{\varepsilon}(x)=\frac{(\mathrm{N}(\mathrm{N}-2) \varepsilon)^{\frac{\mathrm{N}-2}{8}}}{\left(\varepsilon+|x|^{2}\right)^{\frac{N-2}{4}}}, \quad \varepsilon>0$. Note that the function $v_{\varepsilon}=w_{\varepsilon}^{2}$ solves the equation $\Delta v_{\varepsilon}+v_{\varepsilon}^{\mathrm{N}-2}=0$.

Following [5] by direct calculation we work out the terms of $\mathcal{J}\left(t v_{\varepsilon}\right)$ as follows for $0<\alpha<2$.

$$
\begin{aligned}
& \int_{\mathbb{R}^{\mathrm{N}}}\left|\nabla v_{\varepsilon}^{2}\right|^{2} \mathrm{~d} x=\mathrm{S}^{\frac{\mathrm{N}}{2}}+\mathrm{o}\left(\varepsilon^{\frac{\mathrm{N}-2}{2}}\right), \\
& \int_{\mathbb{R}^{\mathrm{N}}}\left|\nabla v_{\mathcal{\varepsilon}}\right|^{2} \mathrm{~d} x=\mathrm{o}\left(\varepsilon^{\frac{\mathrm{N}-2}{4}}|\ln \varepsilon|\right), \\
& \int_{\mathbb{R}^{N}}\left|v_{\varepsilon}\right|^{\alpha}\left|\nabla v_{\varepsilon}\right|^{2} \mathrm{~d} x=o\left(\varepsilon^{\frac{N-2}{8}(2-\alpha)}\right), \\
& \int_{\mathbb{R}^{\mathrm{N}}}\left|\nabla v_{\varepsilon}\right|^{2\left(2^{*}\right)} \mathrm{d} x=\mathrm{S}^{\frac{\mathrm{N}}{2}}+\mathrm{o}\left(\varepsilon^{\frac{\mathrm{N}}{2}},\right. \\
& \int_{\mathbb{R}^{N}} v_{\varepsilon}^{r} \mathrm{~d} x \approx \begin{cases}\varepsilon^{\frac{N}{2}-\frac{1}{8} r(N-2),} & 2^{*}<r<2\left(2^{*}\right), \\
\varepsilon^{\frac{N}{4}}|\ln \varepsilon|, & r=2^{*}, \\
\varepsilon^{\frac{1}{8}} \mathrm{r}(\mathrm{N}-2), & r<2^{*} .\end{cases}
\end{aligned}
$$

Furthermore,

$$
\begin{aligned}
\int_{\mathbb{R}^{N}} \int_{\mathbb{R}^{N}} \frac{\left|v_{\varepsilon}(x)\right|^{p}\left|v_{\varepsilon}(y)\right|^{p}}{|x-y|^{\alpha}} & \geqslant C \varepsilon^{\frac{p(N-2)}{4}} \int_{B_{1}} \int_{B_{1}} \frac{1}{|x-y|^{\alpha}\left(\varepsilon+|x|^{2}\right)^{\frac{p(N-2)}{4}}\left(\varepsilon+|y|^{2}\right)^{\frac{p(N-2)}{4}}} \\
& \geqslant o\left(\varepsilon^{\frac{2 N-\alpha}{2}-\frac{p(N-2)}{4}} \int_{B_{1}} \int_{B_{1}} \frac{1}{|x-y|^{\alpha}\left(1+|x|^{2}\right)^{\frac{p(N-2)}{4}}\left(1+|y|^{2}\right)^{\frac{p(N-2)}{4}}}\right. \\
& =o\left(\varepsilon^{\frac{2 N-\alpha}{2}-\frac{p(N-2)}{4}}\right) .
\end{aligned}
$$

From the previous discussion, therefore, we obtain

$$
\begin{aligned}
\sup _{\mathrm{t} \geqslant 0} \mathcal{J}\left(\mathrm{t} v_{\varepsilon}\right) \leqslant & \mathrm{t}^{4} \int_{\mathbb{R}^{\mathrm{N}}} v_{\varepsilon}^{2}\left|\nabla v_{\varepsilon}\right|^{2} \mathrm{~d} x-\frac{\mathrm{N}-2}{4 \mathrm{~N}} \mathrm{t}^{2\left(2^{*}\right)} \int_{\mathbb{R}^{\mathrm{N}}} v_{\varepsilon}^{2\left(2^{*}\right)} \mathrm{dx} \\
& +\frac{1}{2} \mathrm{t}^{2} \int_{\mathbb{R}^{\mathrm{N}}}\left(\left|\nabla v_{\varepsilon}\right|^{2}+\mathrm{V}_{\infty} v_{\varepsilon}^{2}\right) \mathrm{d} x-\lambda \int_{\mathbb{R}^{\mathrm{N}}} \mathrm{I}_{\alpha} *\left|v_{\varepsilon}\right|^{p}\left|v_{\varepsilon}\right|^{\mathrm{p}} \mathrm{dx} \\
\leqslant & \frac{1}{4} \mathrm{t}^{4} \mathrm{~S}^{\frac{\mathrm{N}}{2}}-\frac{\mathrm{N}-2}{4 \mathrm{~N}} \mathrm{t}^{2\left(2^{*}\right)} \mathrm{S}^{\frac{\mathrm{N}}{2}}+\mathrm{o}\left(\varepsilon^{\frac{\mathrm{N}-2}{4}} \ln \varepsilon\right)-\lambda \mathrm{o}\left(\varepsilon^{\frac{2 \mathrm{~N}-\alpha}{2}-\frac{\mathrm{p}(\mathrm{N}-2)}{4}}\right) \\
\leqslant & \frac{1}{2 \mathrm{~N}} \mathrm{~S}^{\frac{\mathrm{N}}{2}}-\lambda \mathrm{o}\left(\varepsilon^{\frac{2 \mathrm{~N}-\alpha}{2}-\frac{\mathrm{p}(\mathrm{N}-2)}{4}}\right) \\
& <\frac{1}{2 \mathrm{~N}} \mathrm{~S}^{\frac{\mathrm{N}}{2}}
\end{aligned}
$$

Lemma 3.5. Assume that $\left\{w_{n}\right\} \subset \mathrm{E}$ be a bounded $(\mathrm{C})_{\mathrm{c}}$ sequence of $\mathcal{J}$, if $0<\mathrm{c}<\frac{1}{2 \mathrm{~N}} \mathrm{~S}^{\mathrm{N}}$ hold, then there exists a $\zeta>0$ and a sequence $\left\{y_{n}\right\} \subset \mathbb{R}^{N}$ such that $\liminf \int_{B\left(y_{n}\right)} f^{2}\left(w_{n}\right) d x \geqslant \zeta$.

Proof. Assuming that the conclusion is not true, it can be inferred from Lemma 1.21 in [32] that $\mathrm{f}\left(w_{\mathrm{n}}\right) \rightarrow 0$ in $L^{p}\left(\mathbb{R}^{N}\right)$ for all $2<p<2^{*}$. By Lemma 2.1, $\left(\mathrm{A}_{6}\right)$, and the Hardy-Littlewood-Sobolev inequality, we get $\int_{\mathbb{R}^{N}} I_{\alpha} *\left|w_{n}\right|^{p}\left|w_{n}\right|^{p} \mathrm{~d} x \rightarrow 0$ for any $p \in\left(2,2^{*}\right)$. Owing to $c>0$ and $\left\{w_{n}\right\}$ being bounded in $E$. Passing to a subsequence,

$$
\int_{\mathbb{R}^{N}}\left(1+4 f^{2}\left(w_{n}\right)\right)\left(1+2 f^{2}\left(w_{n}\right)^{-1}\left|\nabla w_{n}\right|^{2} d x \rightarrow b\right.
$$

and

$$
\int_{\mathbb{R}^{N}}\left|f\left(w_{n}\right)\right|^{22^{*}} \mathrm{~d} x \rightarrow \mathrm{d}>0
$$


Applying the definition of $S$, we obtain

$$
S\left(\int_{\mathbb{R}^{N}}\left|f\left(w_{n}\right)\right|^{22^{*}} \mathrm{~d} x\right)^{\frac{2}{2^{*}}} \leqslant \int_{\mathbb{R}^{N}}\left|\nabla f^{2}\left(w_{n}\right)\right|^{2} \mathrm{~d} x \int_{\mathbb{R}^{N}}\left(1+4 f^{2}\left(w_{n}\right)\right)\left(1+2 f^{2}\left(w_{n}\right)^{-1}\left|\nabla w_{n}\right|^{2} \mathrm{~d} x,\right.
$$

which yields $S d^{\frac{2}{2^{*}}} \leqslant$ b. Let $w_{n}=\frac{f\left(w_{n}\right)}{f^{\prime}\left(w_{n}\right)}$. Recall that $f\left(w_{n}\right) \rightarrow 0$ in $L^{p+1}\left(\mathbb{R}^{N}\right)$, we get

$$
0=\lim _{n \rightarrow \infty}\left\langle\partial^{\prime}\left(w_{n}\right), w_{n}\right\rangle \geqslant b-d
$$

Hence $d \geqslant S^{\frac{N}{2}}$ and

$$
c=\lim _{n \rightarrow \infty}\left(\mathcal{J}\left(w_{n}\right)-\frac{1}{2}\left\langle\mathcal{J}^{\prime}\left(w_{n}\right), w_{n}\right\rangle \geqslant \lim _{n \rightarrow \infty} \frac{1}{2 N} \int_{\mathbb{R}^{N}}\left|f\left(w_{n}\right)\right|^{22^{*}} d x \geqslant \frac{1}{2 N} S^{\frac{N}{2}},\right.
$$

which contradicts $c<\frac{1}{2 N} S^{\frac{N}{2}}$. The proof is completed.

Proof of Theorem 1.1. Set $\mathrm{c}$ is given by (3.1). From Lemmas 3.1 and 3.2 and the mountain pass theorem, we get that $\mathcal{J}$ has a $(C)_{c}$ sequence $\left\{w_{n}\right\} \subset E$. In the light of (3.2), it is able to hypothesize that $w_{n} \rightarrow w$ in $\mathrm{H}^{1}\left(\mathbb{R}^{\mathrm{N}}\right)$ and $\mathrm{f}\left(w_{n}\right) \rightarrow \mathrm{f}(w)$ in $\mathrm{E}$. It is implies that $\nu_{n} \rightarrow v$ in $\mathrm{L}_{\text {loc }}^{\mathrm{s}}\left(\mathbb{R}^{\mathrm{N}}\right)$ for $2<\mathrm{s}<2^{*}$ and $\mathrm{f}\left(w_{n}\right) \rightarrow \mathrm{f}(w)$ in $\mathrm{L}_{\text {loc }}^{r}\left(\mathbb{R}^{\mathrm{N}}\right)$ for $2<\mathrm{r}\left\langle 22^{*}\right.$, hence $\left\langle\mathcal{J}^{\prime}\left(\mathcal{w}_{n}\right), \varphi\right\rangle \rightarrow\left\langle\mathcal{J}^{\prime}(w), \varphi\right\rangle=0$ for any $\varphi \in \mathrm{C}_{0}^{\infty}\left(\mathbb{R}^{\mathrm{N}}\right)$, that is to say, $v$ is a weak solution of problem (1.1). Furthermore, since the embedding $E \hookrightarrow L^{s}\left(\mathbb{R}^{N}\right)$ is compact for $2 \leqslant s<2^{*}$, we obtain $f\left(w_{n}\right) \rightarrow f(w)$ in $L^{s}\left(\mathbb{R}^{N}\right)$ for $2 \leqslant s<2^{*}$. We can infer from Lemma 3.4 that $c<\frac{1}{2 N} S^{\frac{N}{2}}$, using the Lemma 3.5. There is a constant $\zeta>0$ such that

$$
\int_{\mathbb{R}^{N}} f^{2}(w) d x=\lim _{n \rightarrow \infty} \int_{\mathbb{R}^{N}} f^{2}\left(w_{n}\right) d x \geqslant \zeta .
$$

This implies that $w$ is a nontrivial solution of (1.1). Therefore, $u=f(w)$ is a nontrivial solution of (1.1). In the end, applying $d=: \inf \left\{f(w): w \in E, w \neq 0, J^{\prime}(w)=0\right\}$ and by the lower semicontinuity, we can prove that $d$ is achieved and $u$ is a ground state solution. We complete the proof.

In order to find ground state solutions of problem (1.4), the following limit equation plays a very significant role.

$$
\begin{cases}-\triangle \mathfrak{u}+V(x) u-u \Delta u^{2}=\left(I_{\alpha} *|u|^{p}\right)|u|^{p-2} u+u^{q-2} u, & x \in \mathbb{R}^{N}, \\ u \in H^{1}\left(\mathbb{R}^{N}\right), & x \in \mathbb{R}^{N},\end{cases}
$$

where $\frac{N+\alpha}{N}<p<\frac{N+\alpha}{N-2}, q \in\left(2,2^{*}\right)$, the associated functional is defined as

$$
\mathcal{\partial} v_{\infty}(w)=\frac{1}{2} \int_{\mathbb{R}^{N}}|\nabla w|^{2}+V_{\infty} f^{2}(w)-\frac{1}{2 p} \int_{\mathbb{R}^{N}} I_{\alpha} *|f(w)|^{p}|f(w)|^{p-1} f(w) d x-\left.\frac{1}{q} \int_{\mathbb{R}^{N}}|f(w)|\right|^{q} d x
$$

We define

$$
c_{\infty}=\inf \left\{\mathcal{J}_{\infty}(w) \mid w \in H^{1}\left(\mathbb{R}^{N}\right) \backslash\{0\} \text { and } \mathcal{J}_{V_{\infty}}^{\prime}(w)=0\right\}
$$

Proof of Theorem 1.2. Let us consider the following functional

$$
\partial \mathrm{V}(w)=\frac{1}{2} \int_{\mathbb{R}^{N}}|\nabla w|^{2}+V(x) f^{2}(w)-\frac{1}{2 p} \int_{\mathbb{R}^{N}} I_{\alpha} *|f(w)|^{p}|f(w)|^{p-1} f(w) d x-\frac{1}{q} \int_{\mathbb{R}^{N}}|f(w)|^{q} \mathrm{~d} x,
$$

then critical points of the functional $\partial v(w)$ are weak solution of Eq. (1.4), and vice versa. Similar to the argument in the proofs of Lemmas 3.1 and 3.2, we can conclude that the functional $J_{V}(w)$ also has the mountain pass geometry. Next we give the definition of $c_{V}$, defined by $c_{V}=\inf _{\gamma \in \Gamma_{V}} \max _{t \in[0,1]} \mathcal{J V}(\gamma(t))$, where $\Gamma_{V}=\left\{\gamma \in C[0,1], H^{1}\left(\mathbb{R}^{N}\right) \gamma(0)=1, \mathcal{J V}_{V}(\gamma(1))<0\right\}$. By the definition of $\mathrm{V}$, we obtain $\mathrm{c}_{\mathrm{V}}<\mathrm{c}_{\infty}$. As a matter 
of fact, by Theorem 1.1 the level $c_{\infty}$ is attained at a ground state solution $w_{\infty} \in \mathrm{H}^{1}\left(\mathbb{R}^{\mathrm{N}}\right)$ of the limit problem $\mathcal{J} v_{\infty}(w)$. We can assume without loss of generality that there exists a set of positive measure on which $\mathcal{J}_{v}<\mathcal{J}_{\infty}$ otherwise Theorem 1.2 is a special of Theorem 1.1. We have

$$
c_{V} \leqslant \max _{t \geqslant 0} \mathcal{J} v\left(t w_{\infty}\right)=\mathcal{J} v\left(t_{*} w_{\infty}\right)<\mathcal{J} v_{\infty}\left(t_{*} w_{\infty}\right) \leqslant \max _{t \geqslant 0} \mathcal{J} v_{\infty}\left(t w_{\infty}\right)=c_{\infty}<c,
$$

where $t_{*}>0$ is unique and satisfies that $\left\langle\mathcal{J}^{\prime} v\left(t_{*} \mathcal{W}_{\infty}\right), t_{*} \mathcal{W}_{\infty}\right\rangle=0$. Here, we have taken advantage of the monotonicity of the perturbation, thus the Nehari manifold method works for the detail proofs, we refer to [31]. Let $\left\{w_{n}\right\}$ be a (PS $)_{c_{k}}$ sequence for the functional $\mathcal{J}_{v}(w)$, a standard argument shows that $\left\{w_{n}\right\}$ is bounded in $\mathrm{H}^{1}\left(\mathbb{R}^{N}\right)$ as $n \rightarrow \infty$. Up to a subsequence $w_{n} \rightarrow w$ weakly in $\mathrm{H}^{1}\left(\mathbb{R}^{\mathbb{N}}\right)$ as $n \rightarrow \infty$, and $w_{n}$ converges to $w$ almost everywhere in $\mathbb{R}^{\mathrm{N}}$. By a similar argument as in the proof of Lemma 3.5, we see that there exist $\left\{y_{n}\right\} \in \mathbb{R}^{N}$ and $\delta>0$ such that

$$
\lim _{n \rightarrow \infty} \int_{B_{1}\left(y_{n}\right)}\left|f\left(w_{n}\right)\right|^{2} \geqslant \delta .
$$

We next claim that $\left\{y_{n}\right\}$ is bounded in $\mathbb{R}^{N}$. Thus $f(w)$ is a nontrivial solution of Eq. (1.4). It then follows from Theorem 1.1 similarly, that $\mathcal{J} \mathrm{V}(w) \in\left(0, \mathrm{c}_{\mathrm{k}}\right]$. We now complete this claim indirectly. Suppose that for a subsequence $\left|y_{n}\right| \rightarrow+\infty$ as $n \rightarrow+\infty$, we define $v_{n}=w_{n}\left(\cdot+y_{n}\right)$ and then $\left\{v_{n}\right\}$ is bounded in $H^{1}\left(\mathbb{R}^{N}\right)$, and $v_{n} \rightarrow v \neq 0$. The assumption on the asymptotic shape of the potential $V$ implies that $v$ is a critical point of $\partial v_{\infty}(w)$. In fact, for any $\varphi \in \mathrm{H}^{1}\left(R^{N}\right)$ as $n \rightarrow \infty$, we have

$$
\begin{aligned}
\left|\int_{\Omega}\right| V(x)-V_{\infty}(x)\left|f\left(w_{n}(x)\right)\right| f^{\prime}\left(w_{n}(x)\right) \varphi\left(x-y_{n}\right) d x \mid \\
\leqslant \int_{\frac{B\left(y_{n}\right)}{2}}\left(V(x)-V_{\infty}(x)\right) f\left(\mid w_{n}(x)\right)\left|f^{\prime}\left(w_{n}\right)\right| \varphi\left(x-y_{n}\right) \mid d x \\
\quad+\int_{R^{n} \backslash \frac{B\left(y_{n}\right)}{2}}\left(V(x)-V_{\infty}(x)\right)\left|f\left(w_{n}(x)\right)\right| f^{\prime}\left(w_{n}\right)\left|\varphi\left(x-y_{n}\right)\right| d x \\
\leqslant 2\|V\|_{L^{\infty}}\left\|w_{n}\right\|_{L^{2}}\|\varphi\|_{L^{2}}\left(\mathbb{R}^{n} \backslash \frac{B\left(y_{n}\right)}{2}\right)+\left\|V-V_{\infty}\right\|_{L^{\infty}}\left(\mathbb{R}^{n} \backslash \frac{B\left(y_{n}\right)}{2}\right)\left\|w_{n}\right\|_{L^{2}}\|\varphi\|_{L^{2}} \rightarrow 0 .
\end{aligned}
$$

Since $\frac{B\left(y_{n}\right)}{2}\left(-y_{n}\right) \subset \mathbb{R}^{N} \backslash \frac{B\left(y_{n}\right)}{2}$. Thus, since $v_{n} \rightarrow v$ weakly in $H^{1}\left(\mathbb{R}^{N}\right)$ as $n \rightarrow \infty$, for any $\varphi \in H^{1}\left(\mathbb{R}^{N}\right)$, we obtain

$$
\begin{aligned}
& \left\langle\mathcal{J}^{\prime} \mathrm{V}_{\infty}(v), \varphi\right\rangle=\int_{\mathbb{R}^{N}}|\nabla v||\nabla \varphi|+\mathrm{V}_{\infty} f(v) f^{\prime}(v) \varphi-\int_{\mathbb{R}^{N}} \mathrm{I}_{\alpha} *|f(v)|^{p}|f(v)|^{p-1} f^{\prime}(v) \varphi \mathrm{d} x-\int_{\mathbb{R}^{N}}|f(v)|^{q-1} f^{\prime}(v) \varphi \mathrm{d} x \\
& =\int_{\mathbb{R}^{N}}\left|\nabla v_{n} \| \nabla \varphi\right|+V_{\infty} f\left(v_{n}\right) f^{\prime}\left(v_{n}\right) \varphi-\int_{\mathbb{R}^{N}} I_{\alpha} *\left|f\left(v_{n}\right)\right|^{p}\left|f\left(v_{n}\right)\right|^{p-1} f^{\prime}\left(v_{n}\right) \varphi d x \\
& -\int_{\mathbb{R}^{\mathrm{N}}}\left|\mathbf{f}\left(v_{\mathrm{n}}\right)\right|^{\mathbf{q}-1} \mathbf{f}^{\prime}\left(v_{\mathrm{n}}\right) \varphi \mathrm{d} x \\
& =\left\langle\mathcal{J}^{\prime} \mathrm{v}\left(v_{\mathrm{n}}\right), \varphi\left(x-\mathrm{y}_{\mathrm{n}}\right)\right\rangle+\int_{\mathbb{R}^{\mathrm{N}}}\left(\mathrm{V}(\mathrm{x})-\mathrm{V}_{\infty}\right)\left|\mathrm{f}\left(v_{\mathrm{n}}\right)\right| \mathrm{f}^{\prime}\left(v_{\mathrm{n}}\right) \varphi\left(x-\mathrm{y}_{\mathrm{n}}\right) \mathrm{d} x \\
& =\left\langle\mathcal{J}^{\prime} \vee\left(v_{\mathrm{n}}\right), \varphi\left(x-y_{n}\right)\right\rangle+o_{n}(1) \rightarrow 0 .
\end{aligned}
$$

We can easily deduce that $\mathcal{J}^{\prime} v(v)=0$. However, Fatou's lemma implies that

$$
\begin{aligned}
c_{k}+o_{n}(1)\left\|w_{n}\right\| & =\partial v\left(w_{n}\right)-\frac{1}{2}\left(\mathcal{J}^{\prime} v\left(w_{n}\right), \frac{f\left(w_{n}\right)}{f^{\prime}\left(w_{n}\right)}\right) \\
& \geqslant \frac{1}{2 p} \int_{R^{N}}\left(I_{\alpha} *|f(v)|^{p}\right)|f(v)|^{p}+\left(\frac{1}{2}-\frac{1}{q}\right) \int_{R^{N}} v_{\infty}|f(v)|^{2}+o_{n}(1) \\
& =\partial v_{\infty}(v)-\frac{1}{2}\left\langle\partial^{\prime} v_{\infty}(v), \frac{f(v)}{f^{\prime}(v)}\right\rangle+o_{n}(1) \geqslant c_{\infty}+o_{n}(1) .
\end{aligned}
$$


Therefore, we obtain a contradiction with $c_{k}<c_{\infty}$. Finally, following the strategy of Theorem 1.1, let us consider the following set

$$
\mathrm{m}=\inf \left\{\mathcal{J} v(v)\left|v \in \mathrm{H}^{1}\left(\mathbb{R}^{\mathbb{N}}\right)\right|\{0\} \text { and } \mathcal{J}^{\prime} \mathrm{v}_{\infty}(v)=0\right\} .
$$

Let $\left\{v_{n}\right\}$ be a minimizing sequence for $m$, on the one hand,

$$
m+o_{n}(1)=J V\left(v_{n}\right)=J V\left(v_{n}\right)-\frac{1}{q}\left\langle J_{V}^{\prime}\left(v_{n}\right), \frac{f\left(v_{n}\right)}{f^{\prime}\left(v_{n}\right)}\right\rangle \geqslant\left(\frac{1}{2}-\frac{1}{q}\right)\left\|v_{n}\right\|^{2} .
$$

On the other hand,

$$
\begin{aligned}
\left\|v_{n}\right\|^{2} \leqslant \int_{\mathbb{R}^{N}}\left|\nabla v_{n}\right|^{2}+\mathrm{V}(x) \mathbf{f}^{2}\left(v_{n}\right) & =\int_{\mathbb{R}^{N}} \int_{\mathbb{R}^{N}}\left(I_{\alpha} *\left|f\left(v_{n}\right)\right|^{p}\right)\left|f\left(v_{n}\right)\right|^{p}+\int_{\mathbb{R}^{N}}\left|f\left(v_{n}\right)\right|^{q} \\
& \leqslant C\left(\int_{\mathbb{R}^{N}}\left|v_{n}\right|^{2}\right)^{2 p}+\int_{\mathbb{R}^{N}}\left|v_{n}\right|^{q} \\
& \leqslant C\left\|v_{n}\right\|^{2 p}+C\left\|v_{n}\right\|^{q}
\end{aligned}
$$

We deduce from (3.3) and (3.4) that $0<m \leqslant c_{k}$, and $\left\{v_{n}\right\}_{n \in N}$ is bounded in $H^{1}\left(\mathbb{R}^{N}\right)$. The process is the same as above, $v_{n} \rightarrow v \neq 0$ weakly in $H^{1}\left(\mathbb{R}^{N}\right)$ and $\mathcal{J}^{\prime} v(v)=0$. Applying Fatou's Lemma, we can proof $\partial v(v) \leqslant m$. As a result, we get the ground state solution of $v$ is (1.4).

\section{Acknowledgement}

The authors thank the referees for valuable comments and suggestions which improved the presentation of this manuscript. This work is partially supported by the Fundamental Research Funds for the National Natural Science Foundation of China 11671403 and Guizhou University of Finance and Economics of 2019XYB15.

\section{References}

[1] C. O. Alves, M. Yang, Multiplicity and concentration of solutions for a quasilinear Choquard equation, J. Math. Phys., 55 (2014), 21 pages. 1

[2] C. O. Alves, M. Yang, Multiplicity and concentration behavior of solutions for a quasilinear Choquard equation via penalization method, Proc. Roy. Soc. Edinburgh Sect. A, 146 (2016), 23-58. 1

[3] F. G. Bass, N. N. Nasanov, Nonlinear electromagnetic spin waves, Phys. Rep., 189 (1990), 165-223. 1

[4] A. Borovskii, A. Galkin, Dynamic modulation of an ultrashort high-intensity laser pulse in matter, JETP, 77 (1993), 562-573. 1

[5] H. Brezis, L. Nirengerg, Positive solutions of nonlinear elliptic equations involving critical Sobolev exponents, Comm. Pure Appl. Math., 36 (1983), 437-477. 3

[6] D. Cao, S. J. Peng, A note on the sign-changing solutions to elliptic problems with critical Sobolev and Hardy terms, J. Differential Equations, 193 (2003), 424-434. 1

[7] J. H. Chen, B. Cheng, X. J. Huang, Ground state solutions for a class of quasilinear Schrödinger equations with Choquard type nonlinearity, Appl. Math. Lett., 102 (2020), 7 pages. 1

[8] S. X. Chen, X. Wu, Existence of positive solutions for a class of quasilinear Schrödinger equations of Choquard type, J. Math. Anal. Appl., 475 (2019), 1754-1777. 1

[9] S. Cingolani, M. Clapp, S. Secchi, Multiple solutions to a magnetic nonlinear Choquard equation, Z. Angew. Math. Phys., 63 (2012), 233-248. 1

[10] A. de Bouard, N. Hayashi, J.-C. Saut, Global existence of small solutions to a relativistic nonlinear Schrödinger equation, Comm. Math. Phys., 189 (1997), 73-105. 1

[11] J. M. do Ó, A. Moameni, Solutions for singular quasilinear Schrödinger equations with one parameter, Commun. Pure. Appl. Anal., 9 (2010), 1011-1023. 1

[12] E. Gloss, Existence and concentration of positive solutions for a quasilinear equation in $\mathbb{R}^{\mathrm{N}}$, J. Math. Anal. Appl., 371 (2010), 465-484. 1, 2.1

[13] R. W. Hasse, A general method for the solution of nonlinear soliton and kink Schrödinger equations, Z. Phys. B, 37 (1980), 83-87. 1 
[14] N. Hirano, C. Saccon, N. Shioji, Brezis-Nirenberg type theorems and multiplicity of positive solutions for a singular elliptic problem, J. Differential Equations, 245 (2008), 1997-2037. 1

[15] C. Ji, A. Szulkin, A logarithmic Schrödinger equation with asymptotic conditions on the potential, J. Math. Anal. Appl., 437 (2016), 241-254. 2.2

[16] S. Kurihura, Large-amplitude quasi-solitons in superfluid films, J. Phys. Soc. Japan., 50 (1981), 3262-3267. 1

[17] E. W. Laedke, K. H. Spatschek, L. Stenflo, Evolution theorem for a class of perturbed envelope soliton solutions, J. Math. Phys., 24 (1983), 2764-2769. 1

[18] H. Lange, M. Poppenberg, H. Teismann, Nash-Moser methods for the solution of quasilinear Schrödinger equations, Comm. Partial Differ. Equ., 24 (1999), 1399-1418. 1

[19] H. Lange, B. Toomire, P. F. Zweifel, Time-dependent dissipation in nonlinear Schrodinger systems, J. Math. Phys., 36 (1995), 1274-1283. 1

[20] X. F. Li, S. W. Ma, G. Zhang, Existence and qualitative properties of solutions for Choquard equations with a local term, Nonlinear Anal. Real World Appl., 45 (2019), 1-25.

[21] A. Litvak, A. Sergeev, On one dimensional collapse of plasma waves, JETP Lett., 27 (1978), 517-520. 1

[22] V. Makhankov, V. Fedyanin, Non-linear effects in quasi-one-dimensional models of condensed matter theory, Phys. Rep., 104 (1984), 1-86. 1

[23] V. Moroz, J. Van Schaftingen, Groundstates of nonlinear Choquard equations: existence, qualitative properties and decay asymptotics, J. Funct. Anal., 265 (2013), 153-184. 1

[24] V. Moroz, J. Van Schaftingen, Existence of groundstates for a class of nonlinear Choquard equations, Trans. Amer. Math. Soc., 367 (2015), 6557-6579.

[25] V. Moroz, J. Van Schaftingen, Groundstates of nonlinear Choquard equations Hardy-Littlewood-Sobolev critical exponent, Commun. Contemp. Math., 17 (2015), 12 pages. 1

[26] A. Nakamura, Damping and modification of exciton solitary waves, J. Phys. Soc. Japan, 42 (1977), 1824-1835. 1

[27] M. Poppenberg, K. Schmitt, Z.-Q. Wang, On the existence of soliton solutions to quasilinear Schrödinger equations, Calc. Var. Partial Differ. Equations, 14 (2002), 329-344. 1

[28] V. Radulescu, D. Smets, M. Willem, Hardy-Sobolev inequalities with remainder terms, Topol. Methods Nonlinear Anal., 20 (2002), 145-149.

[29] E. A. B. Silva, G. F. Vieira, Quasilenear asymptotically periodic Schrödinger equations with critical growth, Calc. Var. Partial Differ. Equ., 39 (2010), 1-33.

[30] E. A. B. Silva, G. F. Vieira, Quasilinear asymptotically periodic Schrödinger equations with subcritical growth, Nonlinear Anal., 72 (2010), 2935-2949. 1

[31] A. Szulkin, T. Weth, The method of Nehari manifold, Hand book of nonconvex analysis and applications (Int. Press, Somerville), 2010 (2010), 597-632. 3

[32] M. Willem, Minimax Theorems, Birkhüser Boston, Boston, (1996). 1, 2, 3

[33] X. Y. Yang, W. Zhang, F. Zhao, Existence and muliplicity of solutions for a quasilinear Choquard equation via perturbation method, J. Math. Phys., 59 (2018), 10 pages. 1 